\title{
SMSP-EMOA: Augmenting SMS-EMOA with the Prospect Indicator for Multiobjective Optimization
}

\author{
Dung H. Phan \\ Department of Computer Science \\ University of Massachusetts, Boston \\ Boston, MA 02125 \\ phdung@cs.umb.edu
}

\author{
Junichi Suzuki \\ Department of Computer Science \\ University of Massachusetts, Boston \\ Boston, MA 02125 \\ jxs@cs.umb.edu
}

\author{
Pruet Boonma \\ Department of Computer Engineering \\ Chiang Mai University \\ Chiang Mai, 50200, Thailand \\ pruet@eng.cmu.ac.th
}

\begin{abstract}
This paper studies a new evolutionary multiobjective optimization algorithm (EMOA) that leverages quality indicators in parent selection and environmental selection operators. The proposed indicator-based EMOA, called SMSPEMOA, is designed as an extension to SMS-EMOA, which is one of the most successfully and widely used indicatorbased EMOAs. SMSP-EMOA uses the prospect indicator in its parent selection and the hypervolume indicator in its environmental selection. The prospect indicator measures the potential (or prospect) of each individual to reproduce offspring that dominate itself and spread out in the objective space. It allows the parent selection operator to (1) maintain sufficient selection pressure, even in high dimensional MOPs, thereby improving convergence velocity toward the Pareto-optimal front, and (2) diversify individuals, even in high dimensional MOPs, thereby spread- ing out individuals in the objective space. Experimental results show that SMSP-EMOA's parent selection operator complement its environmental selection operator. SMSP-EMOA outperforms SMS-EMOA and well-known traditional EMOAs in optimality and convergence velocity without sacrificing the diversity of individuals.
\end{abstract}

Keywords-Evolutionary multiobjective optimization algorithms (EMOAs), Quality indicators, Indicator-based EMOAs

\section{INTRODUCTION}

This paper proposes and evaluates a new indicator-based evolutionary algorithm to solve multiobjective optimization problems (MOPs). An MOP is described as follows:

$$
\left.\begin{array}{r}
\operatorname{minimize} F(\vec{x})=\left[f_{1}(\vec{x}), f_{2}(\vec{x}), \cdots, f_{n}(\vec{x})\right]^{T} \in \mathcal{O} \\
\text { subject to } \vec{x}=\left[x_{1}, x_{2}, \cdots, x_{m}\right]^{T} \in \mathcal{S}
\end{array}\right\}
$$

$\mathcal{S}$ denotes the decision variable space. $\vec{x} \in \mathcal{S}$ denotes a solution candidate that consists of $m$ decision variables. It is called an individual in evolutionary multiobjective optimization algorithms (EMOAs). $F$ consists of $n$ real-value objective functions, which produce the objective values of $\vec{x}$ in the objective space $\mathcal{O}$. When $m \geq 3$, an MOP is called high-dimensional [1]. The goal of an EMOA is to find an individual(s) that minimize(s) objective values.

In MOPs, there rarely exists a single solution that is optimum with respect to all objectives because objective functions conflict with each other. Thus, EMOAs seek the optimal trade-off individuals, or Pareto-optimal individuals, by considering the trade-offs among conflicting objectives. The notion of dominance plays an important role to seek Pareto optimality [2]. An individual $\vec{x} \in \mathcal{S}$ is said to dominate another individual $\vec{y} \in \mathcal{S}$ (denoted by $\vec{x} \succ \vec{y}$ ) iif the both of the following conditions are hold:

- $f_{i}(\vec{x}) \leq f_{i}(\vec{y}) \forall i=1, \cdots, n$

- $f_{i}(\vec{x})<f_{i}(\vec{y}) \exists i=1, \cdots, n$

EMOAs often rank individuals based on the dominance relationships among them and exploit their ranks in selection operators [2]. This process is called dominance ranking.

A research trend in the design space of EMOAs is to adopt indicator-based selection operators based on quality indicators, which are designed to augment or replace dominance ranking [3]. A quality indicator measures the goodness of each individual. For example, the hypervolume indicator [4], or the S metric, and its variants [5]-[9] have been used in various EMOAs' selection operators. Recent research findings (e.g., [10]) show that indicator-based EMOAs outperform traditional EMOAs that use dominance ranking. SMS-EMOA (S Metric Selection EMOA) [11] is one of the most successfully and widely used indicator-based EMOAs [10], [12]-[17]. It uses the hypervolume indicator in its environmental selection operator, which chooses a set of individuals used in the next generation from the union of the current population and its offspring.

This paper extends SMS-EMOA with a quality indicator, called prospect indicator. The prospect indicator measures the potential (or prospect) of each individual to reproduce offspring that dominate itself and spread out in the objective space. The proposed EMOA, SMSP-EMOA (S Metric Selection with the Prospect indicator), uses the prospect indicator in its parent selection operator, which chooses parent individuals from the population to reproduce offspring, as well as the hypervolume indicator in its environmental selection operator. The prospect indicator allows the parent selection operator to (1) maintain sufficient selection pressure, even in high dimensional MOPs, thereby improving convergence 
velocity toward the Pareto-optimal front, and (2) diversify individuals, even in high dimensional MOPs, thereby spreading out individuals in the objective space.

Experimental results show that SMSP-EMOA's parent selection operator complement its environmental selection operator. SMSP-EMOA outperforms SMS-EMOA and wellknown traditional EMOAs (NSGA-II [18] and SPEA2 [19]) in optimality and convergence velocity without sacrificing the diversity of individuals.

\section{RELATED WORK}

SMSP-EMOA extends SMS-EMOA's parent selection operator with the prospect indicator. While SMS-EMOA uses the hypervolume indicator for its environmental selection, it uses no indicators for its parent selection. Instead, it randomly draws two parent individuals from the population [11], [20]. SMSP-EMOA performs a binary tournament with the prospect indicator for parent selection.

The hypervolume indicator and its variants [4]-[9] are similar to the prospect indicator in that both are volumebased indicators. The hypervolume indicator measures the volume of a hypercube that each individual dominates in the objective space. The hypercube is formed with the individual and a reference point representing the highest (or worst) possible objective values. In contrast, the prospect indicator measures the volume of a hypercube in the opposite way. It considers a hypercube that dominates an individual. The hypercube is formed with the individual and the Utopian point, which represents the lowest (or best) possible objective values. The Utopian point is the $(0,0)$ point in a two dimensional objective space. While the hypervolume indicator requires a carefully chosen reference point depending on an MOP to solve [5], [21], it is always trivial to choose the Utopian point for the prospect indicator regardless of MOPs.

SPAM is similar to SMSP-EMOA in that it uses multiple indicators as SMSP-EMOA does [8]. It can use two or more indicators in its environmental selection operator. Unlike SPAM, SMSP-EMOA uses different indicators (i.e., the prospect and hypervolume indicators) in different selection operators (i.e., parent selection and environmental selection operators). This paper is the first attempt to investigate how two different volume-based indicators interact and complement with each other across different selection operators.

H-SMS-EMOA is similar to SMSP-EMOA in that both algorithms extend SMS-EMOA. It combines SMS-EMOA and local search algorithms in two ways: relay, which executes local search after SMS-EMOA's evolutionary process is over, and hybridization, which embeds local search within SMS-EMOA [12]. SMSP-EMOA extends SMS-EMOA by introducing an extra indicator in parent selection rather than introducing extra search algorithms.

There are several studies to reduce the computational cost of SMS-EMOA. Klinkenberg et al. attempt it with adaptive mutation, kriging and steady-state parallelization [13].
Ishibuchi et al. use scalarizing functions to approximate the hypervolume contribution of an individual [17]. SMSPEMOA does not focus on cost reduction for SMS-EMOA.

Naujoks et al. propose an online statistical technique to determine the termination condition (i.e., the maximum number of objective function evaluations) for SMSEMOA [16]. SMSP-EMOA does not focus on dynamic adjustment of the termination condition, but uses staticallyfixed termination conditions.

\section{SMSP-EMOA}

This section describes SMSP-EMOA's algorithmic structure and its operators.

\section{A. Algorithmic Structure}

Algorithm 1 shows SMSP-EMOA's algorithmic structure, which extends SMS-EMOA's.

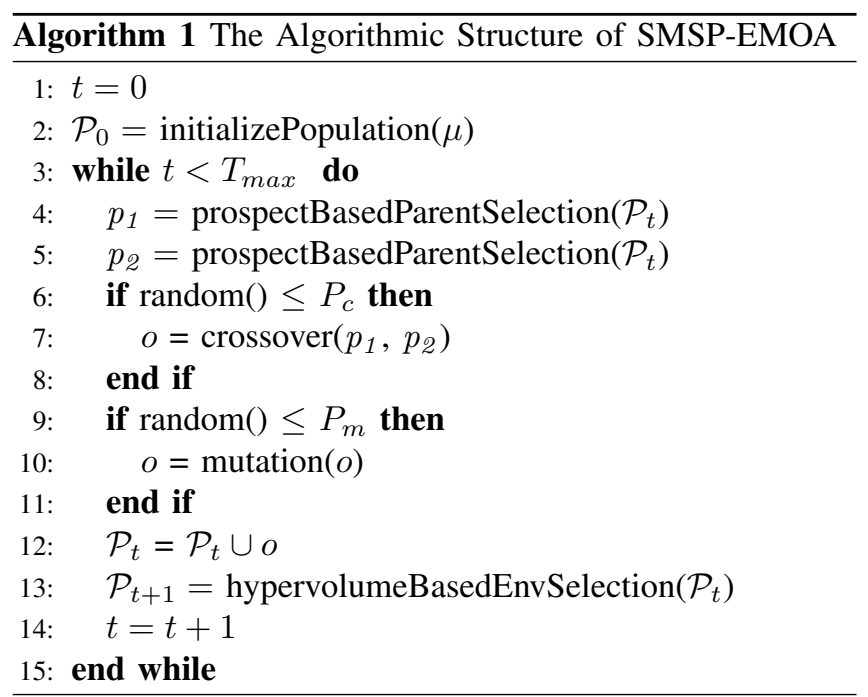

In the first iteration $(t=0), \mu$ individuals are randomly generated as the initial population $P_{0}$ (Line 2). In each iteration $(t)$, a pair of individuals, called parents $\left(p_{1}\right.$ and $\left.p_{2}\right)$, are chosen from the current population $\mathcal{P}_{t}$ with the proposed parent selection operator, which uses the prospect indicator (prospectBasedParentSelection(), Lines 4 and 5).

With the crossover rate $P_{c}$, two parents reproduce one offspring with the SBX (self-adaptive simulated binary crossover) operator [22] (Lines 6 and 7). Polynomial mutation [18] is performed on the offspring with the mutation rate of $P_{m}$ (Lines 9 to 10). The offspring is combined with the population $\mathcal{P}_{t}$ to form a pool of candidate individuals used in the next iteration $(t=t+1)$.

Environmental selection follows reproduction. One individual is eliminated from $\mathcal{P}_{t}$ to produce $\mathcal{P}_{t+1}$ (hypervolumeBasedEnvSelection(), Line 13). Environmental selection performs a $(\mu+1)$ steady state evolution. 


\section{B. Parent Selection with the Prospect Indicator}

Algorithm 2 shows how the proposed parent selection operator (prospectBasedParentSelection() in Algorithm 1) works with the prospect indicator. It is designed as a variant of binary tournament selection. It randomly draws two individuals from the current population $\mathcal{P}$, compares them based on the dominance relationship between them and chooses a superior one as a parent (Lines 5 to 8). Note that $p_{1} \succ p_{2}$ means $p_{1}$ dominates $p_{2}$ as described in Section I.

If two individuals $\left(p_{1}\right.$ and $\left.p_{2}\right)$ do not dominate each other and are placed in the same rank, the proposed selection operator chooses one of them as a parent with the prospect indicator. Lines 10 and 11 compute the prospect indicator values of $p_{1}$ and $p_{2}\left(I_{P}\left(p_{1}\right)\right.$ and $\left.I_{P}\left(p_{2}\right)\right)$, and Line 12 compares the two values. The proposed operator chooses the one with a higher $I_{P}$ value (Lines 12 to 16).

The prospect indicator value of an individual $i\left(I_{P}(i)\right)$ is computed as follows:

$$
I_{P}(i)=V\left(\mathcal{R}_{\operatorname{rank}(i)}\right)-V\left(\mathcal{R}_{\operatorname{rank}(i)} \backslash\{i\}\right)
$$

$\operatorname{rank}(i)$ denotes the value of a rank that $i$ is placed at. $\mathcal{R}_{\text {rank(i) }}$ denotes a set of individuals that are placed at the rank of $\operatorname{rank}(i) . \mathcal{R}_{1}$ contains the individuals of the best (or highest) rank (i.e., the non-dominated individuals in $\mathcal{P}) . \mathcal{R}_{2}$ contains the individuals of the second highest rank (i.e., individuals that are non-dominated in $\mathcal{P}_{t} \backslash \mathcal{R}_{1}$ ). $V(\mathcal{R})$ denotes the volume of a hypercube that dominates the individuals in $\mathcal{R}$ in the objective space. It is calculated with the Lebesgue measure as follows.

$$
V(\mathcal{R})=\Lambda\left(\bigcup_{x \in \mathcal{R}}\left\{x^{\prime} \mid x_{u} \succ x^{\prime} \succ x\right\}\right)
$$

$x_{u}$ denotes the Utopian point, and $\Lambda$ denotes the Lebesgue measure.

The prospect indicator valuates the potential (or prospect) of an individual to reproduce offspring that dominate itself. Figure 1 shows an example measurement of the prospect indicator in a two dimensional objective space. This example considers three non-dominated individuals: $a, b$ and $c\left(\mathcal{R}_{\text {rank }(a)}=\mathcal{R}_{\operatorname{rank}(b)}=\mathcal{R}_{\operatorname{rank}(c)}=\{a, b, c\}\right)$. The Utopian point is $(0,0) . I_{P}(b)$ is a shaded area in Figure 1 (i.e., $V\left(\mathcal{R}_{\text {rank(b) }}\right)-V\left(\mathcal{R}_{\operatorname{rank}(b)} \backslash\{b\}\right)$ ).

Algorithm 3 shows how to compute $I_{P}(p)$. $\mathcal{P}$ denotes a set of individuals that are placed at the same rank as an individual $p$. For each objective (o), the distance between $p$ and $s$ is measured to compute $I_{P}(p)$, where $s$ denotes an individual that yields the closest yet superior objective value.

\section{Environmental Selection with the Hypervolume Indicator}

Algorithm 4 shows how environmental selection (hypervolumeBasedEnvSelection() in Algorithm 1) works with the hypervolume indicator. In environmental
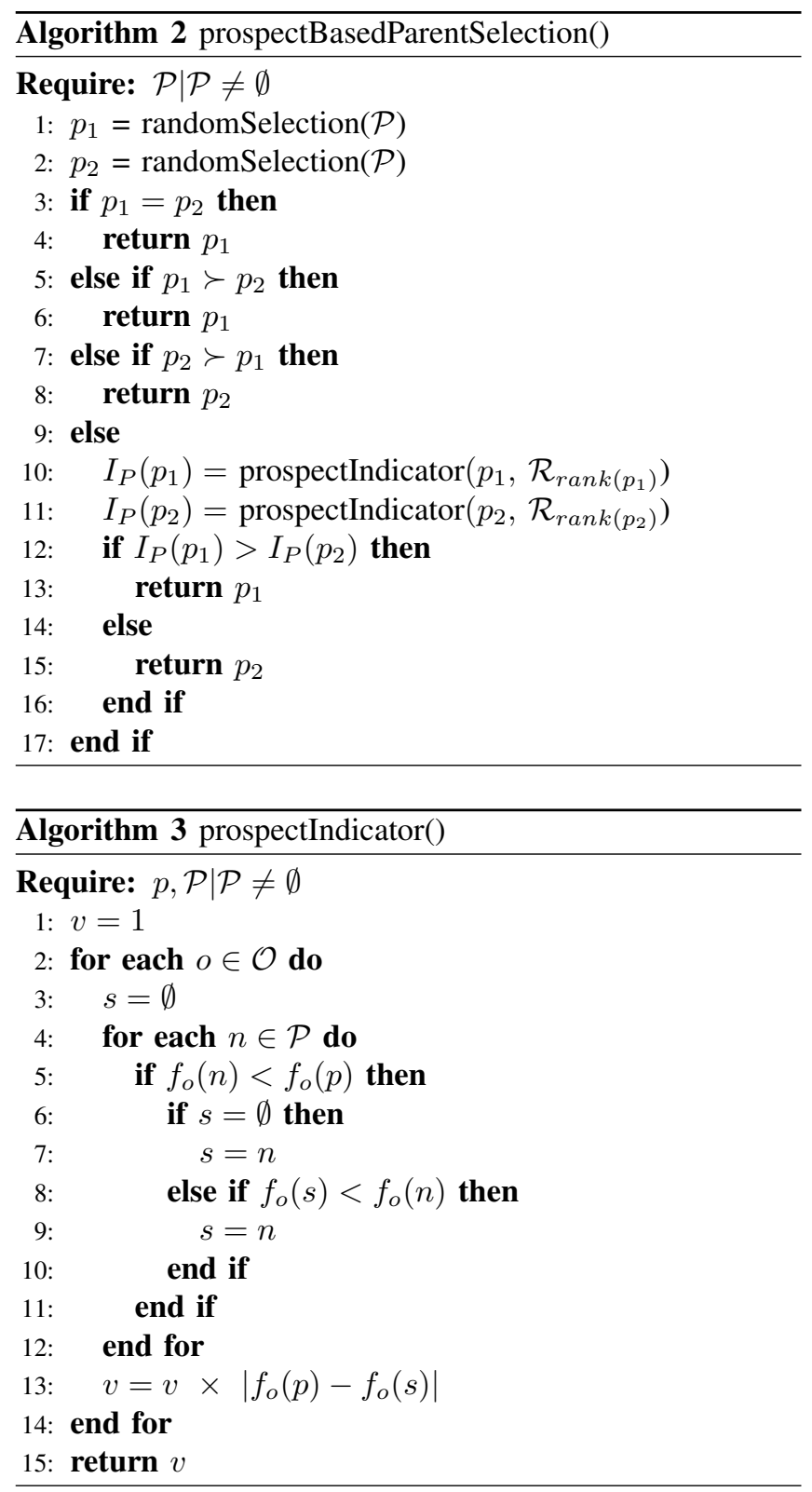

selection, $\mu$ individuals are selected from $\mu+1$ individuals as the population used in the next iteration.

dominanceRanking () performs dominance ranking on the current population $\mathcal{P}$ (Line 1 ). $\mathcal{R}_{1}$ and $\mathcal{R}_{v}$ contain the best-ranked and worst-ranked individuals, respectively. In Lines 2 and 3 , an individual $p$ is discarded from $\mathcal{R}_{v} . p$ is an individual that yields the minimum value of exclusive hypervolume contribution $I_{H} . I_{H}$ of an individual $i \in \mathcal{R}_{v}$ is computed as follows:

$$
I_{H}(i)=H\left(\mathcal{R}_{v}\right)-H\left(\mathcal{R}_{v} \backslash\{i\}\right)
$$

$H\left(\mathcal{R}_{v}\right)$ denotes the volume of a hypercube that the worst-ranked individuals dominate. It is calculated with the 


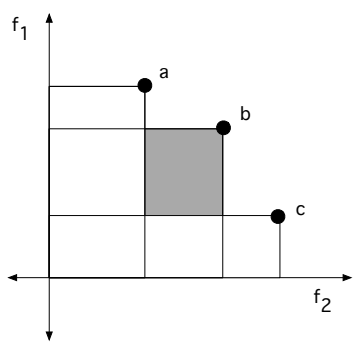

Figure 1: An Example Measurement of Prospect Indicator

Lebesgue measure as follows.

$$
H\left(\mathcal{R}_{v}\right)=\Lambda\left(\bigcup_{x \in \mathcal{R}_{v}}\left\{x^{\prime} \mid x \succ x^{\prime} \succ x_{r}\right\}\right)
$$

$x_{r}$ denotes a reference point in the objective space. Figure 2 shows an example measurement of $I_{H}$ in a two dimensional objective space. This example considers three individuals in $\mathcal{R}_{v}: a, b$ and $c . x_{r}=\left(r_{1}, r_{2}\right) . I_{H}(b)$ is a shaded area in Figure 2 (i.e., $H\left(\mathcal{R}_{v}\right)-H\left(\mathcal{R}_{v} \backslash\{b\}\right)$ ).
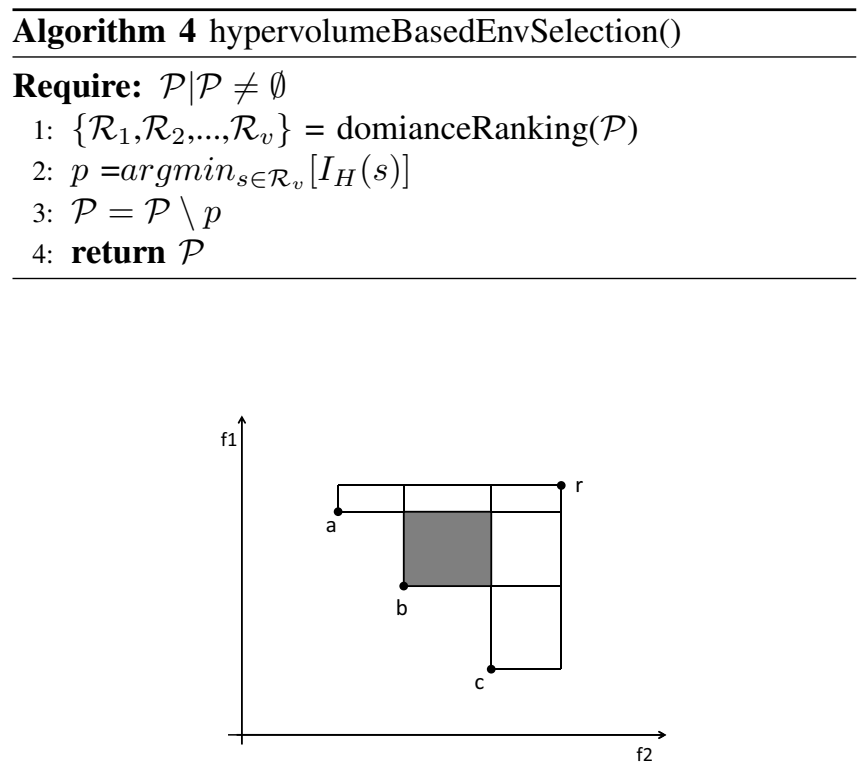

Figure 2: An Example Measurement of Exclusive Hypervolume Contribution

\section{EXPERIMENTAL RESUlts}

This section evaluates SMSP-EMOA with five ZDT family problems [23] and four DTLZ family problems [24]. This section also compares SMSP-EMOA with SMS-EMOA and two classical EMOAs that use dominance ranking for selection: NSGA-II [18] and SPEA2 [19].

\section{A. Experimental Configurations}

SMSP-EMOA was configured as shown in Table I. SMSEMOA, NSGA-II and SPEA2 were configured as described in [11], [18] and [19], respectively. All experiments were conducted with jMetal [20]. Each experimental result is the average of 20 independent results.

Table I: Experimental Configurations

\begin{tabular}{|c|c||c|c|}
\hline Parameter & Value & Parameter & Value \\
\hline \hline$\mu$ (Algo. 1) & 100 & $m$ (ZDT4, Eq. 1) & 10 \\
$T_{\max }$ (ZDT1, Algo. 1) & 15,000 & $m$ (ZDT6, Eq. 1) & 10 \\
$T_{\max }$ (ZDT2, Algo. 1) & 15,000 & $m$ (DTLZ1, Eq. 1) & 7 \\
$T_{\max }$ (ZDT3, Algo. 1) & 15,000 & $m$ (DTLZ2, Eq. 1) & 12 \\
$T_{\max }$ (ZDT4, Algo. 1) & 15,000 & $m$ (DTLZ3, Eq. 1) & 12 \\
$T_{\max }$ (ZDT6, Algo. 1) & 15,000 & $m$ (DTLZ4, Eq. 1) & 12 \\
$T_{\max }$ (DTLZ1, Algo. 1) & 20,000 & Crossover operator & $\mathrm{SBX}$ \\
$T_{\max }$ (DTLZ2, Algo. 1) & 20,000 & $P_{c}$ (Algo. 1) & 0.9 \\
$T_{\max }$ (DTLZ3, Algo. 1) & 50,000 & Mutation operator & Polynomial \\
$T_{\max }$ (DTLZ4, Algo. 1) & 50,000 & $P_{m}$ (Algo. 1) & $1 / m$ \\
$m$ (ZDT1, Eq. 1) & 30 & & \\
$m$ (ZDT2, Eq. 1) & 30 & & \\
$m$ (ZDT3, Eq. 1) & 30 & & \\
\hline
\end{tabular}

\section{B. Evaluation Metrics}

This paper uses three evaluation metrics: hypervolume ratio (HVR), generational distance (GD) and inverted generational distance (IGD).

HVR is calculated as the ratio of the hypervolume $(H V)$ of non-dominated individuals $(D)$ to the hypervolume of Pareto-optimal solutions $\left(P^{*}\right)$ [25].

$$
H V R(D)=\frac{H V(D)}{H V\left(P^{*}\right)}
$$

$H V$ measures the union of the volumes that a given set of individuals dominate [4]. Thus, HVR quantifies the optimality and diversity of non-dominated individuals $(D)$. A higher HVR indicates that non-dominated individuals are closer to the Pareto-optimal front and more diverse in the objective space.

GD is computed as follows where $d\left(d_{i}, P^{*}\right)$ denotes the minimum distance from a non-dominated individual $d_{i}$ to the closest Pareto-optimal solution in the objective space [26].

$$
G D(D)=\sum_{i=1}^{|D|} \frac{d\left(d_{i}, P^{*}\right)}{|D|}
$$

GD measures the optimality of non-dominated individuals $(D)$. A lower GD indicates that non-dominated individuals are closer to the Pareto-optimal front.

IGD is computed as follows where $d\left(z_{i}, D\right)$ denotes the minimum distance from a Pareto-optimal solution $z_{i}$ to the closest non-dominated individual in the objective space [26].

$$
\operatorname{IGD}(D)=\sum_{i=1}^{\left|P^{*}\right|} \frac{d\left(z_{i}, D\right)}{\left|P^{*}\right|}
$$


IGD measures the optimality and diversity (more specifically, extent) of non-dominated individuals $(D)$. A lower IGD indicates that non-dominated individuals are closer to the Pareto-optimal front and their extent is wider.

In order to measure HVR, GD and IGD, $P^{*}$ are taken uniformly from the Pareto-optimal front. $\left|P^{*}\right|=1,001$, 1,001, 269, 1,001, 1,001, 10,000, 10,000, 4,000 and 4,000 in ZDT1, ZDT2, ZDT3, ZDT4, ZDT6, DTLZ1, DTLZ2, DTLZ3 and DTLZ4. This is the default setting in jMetal.

\section{Optimality and Diversity Analysis}

This section evaluates the optimality and diversity of individuals with HVR, GD and IGD. Table II shows the average HVR, GD and IGD values that SMSP-EMOA and other three algorithms yield at the last function evaluation ( $T_{\max }$. See Table I and Algorithm 1.) in ZDT problems. A value in parentheses indicates a standard deviation. A bold number indicates the best result among four algorithms. A double star $(* *)$ or a single star $(*)$ is placed for an average result when the result is significantly different from SMSPEMOA's result based on a single-tail $t$-test. A double star is placed with the confidence level of $99 \%$ while a single star is placed with the confidence level of $95 \%$.

SMSP-EMOA yields the best average HVR among four algorithms in all ZDT problems. In ZDT1, ZDT2 and ZDT6, SMSP-EMOA significantly outperforms the other three algorithms in HVR with the confidence level of $99 \%$. SMSP-EMOA significantly outperforms SMS-EMOA in all ZDT problems except ZDT3. It significantly outperforms SPEA2 in all ZDT problems.

SMSP-EMOA yields the best average GD among four algorithms in ZDT4 and ZDT6. In ZDT6, it significantly outperforms the other three algorithms in GD with the confidence level of $99 \%$. SMSP-EMOA significantly outperforms SMS-EMOA in ZDT3, ZDT4 and ZDT6.

In IGD, SMSP-EMOA yields the best average results in ZDT1 and ZDT6. In ZDT6, it significantly outperforms the other three algorithms in IGD with the confidence level of 99\%. SMSP-EMOA outperforms SMS-EMOA in all ZDT problems.

Table III shows the average HVR, GD and IGD values that SMSP-EMOA and other three algorithms yield at the last function evaluation ( $T_{\max }$. See Table I and Algorithm 1.) in DTLZ problems, each of which has three dimensional objective space. SMSP-EMOA yields the best average HVR among four algorithms in all ZDT problems except DTLZ2 in which SMSP-EMOA is the second best. It significantly outperforms SPEA2 in all ZDT problems.

SMSP-EMOA is not as good as SMS-EMOA in GD; however, its IDG is better than SMS-EMOA's in all DTLZ problems except DTLZ2 in which its IGD is equal to SMSEMOA's.

It is noticeable that standard deviations are high in ZDT4, DTLZ3 and DTLZ4. Therefore, Figure 3 shows the boxplots for those problems to examine the degree of dispersion and skewness in HVR, GD and IGD of all the individuals obtained through 20 independent experiments $(20,000$ individuals in total). A box in each boxplot contains the middle $50 \%$ of individuals. The upper edge of the box indicates the 75th percentile of individuals, and the lower edge indicates the 25th percentile. The middle horizontal line in the box indicates the 50th percentile (i.e., the median). The ends of a vertical line indicate the maximum and minimum individuals.

Figure 3(a) to (c) depict the box plots for HVR, GD and IGD in ZDT4. In ZDT4, SMSP-EMOA yields the best or second best median values in all three metrics. SMSPEMOA also maintains the smallest box in each of Figure 3(a) to (c). This means that SMSP-EMOA achieves the lowest dispersion and skewness in HVR, GD and IGD of individuals. SMSP-EMOA yields more stable performance than the other three algorithms. Qualitatively similar observations can be made in DTLZ3 (Figure 3(d) to (f)) and DTLZ4 (Figure 3(g) to (i)).

Table II: HVR, GD and IGD in ZDT problems

\begin{tabular}{|c|c|c|c|c|}
\hline Problem & Algorithm & HVR & GD & IGD \\
\hline \multirow{4}{*}{ ZDT1 } & NSGAII & $0.98269(0.00128)^{* * *}$ & 5.1E-4(9.0E-5)** & $2.4 \mathrm{E}-4(1.0 \mathrm{E}-5)^{* * *}$ \\
\hline & SPEA2 & $0.97726(0.00313) * *$ & $8.4 \mathrm{E}-4(1.7 \mathrm{E}-4) * *$ & $2.9 \mathrm{E}-4(4.0 \mathrm{E}-5)^{* *}$ \\
\hline & SMS-EMOA & $0.99048(6.5 \mathrm{E}-4)^{* *}$ & $2.0 \mathrm{E}-4(7.0 \mathrm{E}-5) * *$ & $1.49 \mathrm{E}-4(0.0)^{* * *}$ \\
\hline & SMSP-EMOA & $0.99123(6.7 \mathrm{E}-4)$ & $0.00212(6.7 \mathrm{E}-4)$ & $1.46 \mathrm{E}-4(0.0)$ \\
\hline \multirow{4}{*}{ ZDT2 } & NSGAII & $0.95847(0.00446)^{* * *}$ & $6.4 \mathrm{E}-4(1.1 \mathrm{E}-4)$ & $2.8 \mathrm{E}-4(3.0 \mathrm{E}-5)$ \\
\hline & SPEA2 & $0.81407(0.16344) * *$ & $0.00118(2.4 \mathrm{E}-4)^{* *}$ & $0.00349(0.00396)^{* * *}$ \\
\hline & SMS-EMOA & $0.91286(0.09076)^{* * *}$ & 2.3E-4(9.0E-5)** & $0.00206(0.00239)^{* *}$ \\
\hline & SMSP-EMOA & $0.9771(0.0158)$ & $5.3 \mathrm{E}-4(4.4 \mathrm{E}-4)$ & $3.7 \mathrm{E}-4(6.0 \mathrm{E}-4)$ \\
\hline \multirow{4}{*}{ ZDT3 } & NSGAII & $0.98785(0.00206)^{* * *}$ & 3.4E-4(6.0E-5)** & 3.1E-4(2.0E-5)* \\
\hline & SPEA2 & $0.97941(0.00271)^{* * *}$ & $4.9 \mathrm{E}-4(8.0 \mathrm{E}-5)^{* *}$ & 6.4E-4(0.00105) \\
\hline & SMS-EMOA & $0.98882(0.01754)$ & $3.8 \mathrm{E}-4(7.8 \mathrm{E}-4)^{* *}$ & $0.00141(0.00214)$ \\
\hline & SMSP-EMOA & $0.99465(0.00268)$ & $0.00145(6.5 \mathrm{E}-4)$ & $0.00118(0.00201)$ \\
\hline \multirow{4}{*}{ ZDT4 } & NSGAII & $0.8773(0.09757)$ & $0.00512(0.00555)$ & 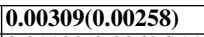 \\
\hline & SPEA2 & $0.57155(0.24622)^{* * *}$ & $0.0388(0.06792)^{*}$ & $0.01132(0.00626)^{* *}$ \\
\hline & \begin{tabular}{|l} 
SMS-EMOA \\
\end{tabular} & $0.71216(0.23092) * *$ & $0.02232(0.04321)^{*}$ & $0.00946(0.00561)^{* *}$ \\
\hline & SMSP-EMOA & $0.90369(0.10046)$ & $0.0042(0.00365)$ & $0.00386(0.0035)$ \\
\hline \multirow{4}{*}{ ZDT6 } & NSGAII & $0.79989(0.03726)^{* * *}$ & $0.00617(0.0013)^{* *}$ & $0.00262(6.4 \mathrm{E}-4)^{* *}$ \\
\hline & SPEA2 & $0.666(0.05061) * *$ & $0.01097(0.00182)^{* * *}$ & $0.00466(8.0 \mathrm{E}-4)^{* *}$ \\
\hline & \begin{tabular}{|l} 
SMS-EMOA \\
\end{tabular} & $0.85502(0.02427)^{* * *}$ & $0.00435(7.6 \mathrm{E}-4)^{* *}$ & $0.00181(3.3 \mathrm{E}-4) * *$ \\
\hline & SMSP-EMOA & $0.9407(0.00876)$ & $0.00177(2.5 \mathrm{E}-4)$ & $7.1 \mathrm{E}-4(1.2 \mathrm{E}-4)$ \\
\hline
\end{tabular}

Table III: HVR, GD and IGD in DTLZ problems

\begin{tabular}{|c|c|c|c|c|}
\hline Problem & Algorithm & HVR & GD & IGD \\
\hline \multirow{4}{*}{ DTLZ1 } & NSGAII & $0.4912(0.35931)^{* * *}$ & $0.25726(0.36029)$ & $0.00409(0.00439)^{* * *}$ \\
\hline & SPEA2 & $0.79465(0.24123)^{* * *}$ & $0.42121(0.55926)^{*}$ & $0.00144(0.0017)^{* *}$ \\
\hline & SMS-EMOA & $0.93192(0.06896)$ & $0.06058(0.1779)^{*}$ & $5.4 \mathrm{E}-4(3.5 \mathrm{E}-4)$ \\
\hline & SMSP-EMOA & $0.94748(0.00946)$ & $0.14178(0.123)$ & $4.6 \mathrm{E}-4(4.0 \mathrm{E}-5)$ \\
\hline \multirow{4}{*}{ DTLZ2 } & NSGAII & 0.80359 & $0.0014(1.9 \mathrm{E}-4) * *$ & $7.6 \mathrm{E}-4(3.0 \mathrm{E}-5) * *$ \\
\hline & SPEA2 & $0.86276(0.00486)^{* *}$ & $0.0013(1.3 \mathrm{E}-4)^{* *}$ & $5.9 \mathrm{E}-4(1.0 \mathrm{E}-5) * *$ \\
\hline & SMS-EMOA & $0.91111(5.6 \mathrm{E}-4) * *$ & 7.4E-4(5.0E-5)* & $8.4 \mathrm{E}-4(1.0 \mathrm{E}-5)$ \\
\hline & $\overline{\text { SMSP-EMOA }}$ & $0.90912(8.5 \mathrm{E}-4)$ & $7.9 \mathrm{E}-4(9.0 \mathrm{E}-5)$ & $8.4 \mathrm{E}-4(2.0 \mathrm{E}-5)$ \\
\hline \multirow{4}{*}{ DTLZ3 } & NSGAII & $0.67004(0.16498)^{* *}$ & $0.01218(0.02251)^{* * *}$ & $0.00224(0.00336)^{* *}$ \\
\hline & SPEA2 & $0.66784(0.249)^{* *}$ & $0.37799(0.68953)^{* * *}$ & $0.00289(0.0042)^{* *}$ \\
\hline & SMS-EMOA & $0.88058(0.05345)$ & $0.00527(0.01074) * *$ & $0.00137(2.5 \mathrm{E}-4)$ \\
\hline & SMSP-EMOA & $0.89234(0.01355)$ & $0.0766(0.05116)$ & $0.00133(3.0 \mathrm{E}-5)$ \\
\hline \multirow{4}{*}{ DTLZ4 } & NSGAII & $0.89177(0.01171)$ & $0.00509(2.2 \mathrm{E}-4)^{*}$ & 0.00119(9.0E-5)** \\
\hline & SPEA2 & $0.76659(0.23025)^{*}$ & $0.00457(7.1 \mathrm{E}-4) *$ & $0.00397(0.00294)$ \\
\hline & SMS-EMOA & $0.8122(0.24977)$ & $0.004889(0.00144)^{*}$ & $0.00279(0.002589)$ \\
\hline & $\overline{\text { SMSP-EMOA }}$ & $\overline{0.91789(0.18862)}$ & $0.005626(0.00114)$ & $0.001977(0.0024904$ \\
\hline
\end{tabular}




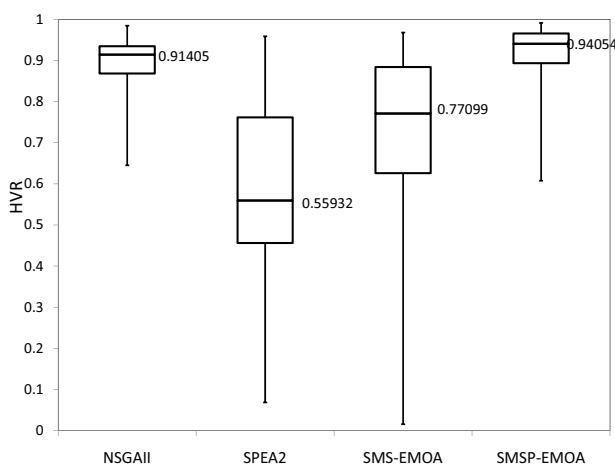

(a) HVR in ZDT4

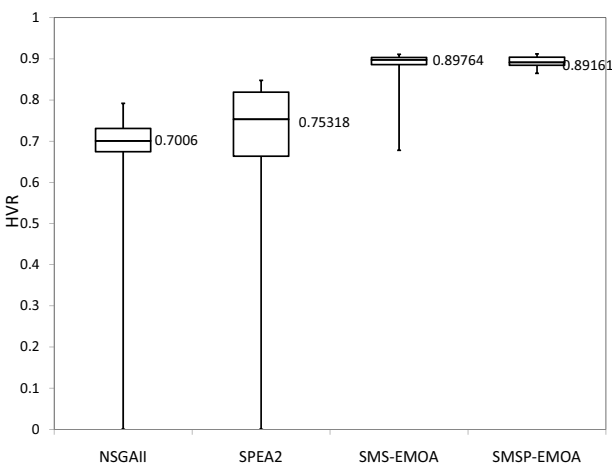

(d) HVR in DTLZ3

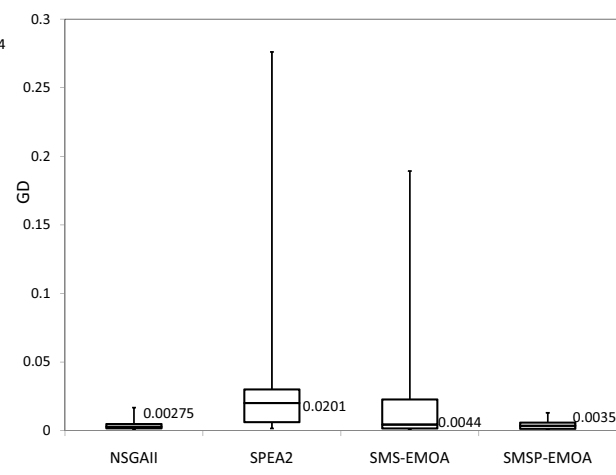

(b) GD in ZDT4

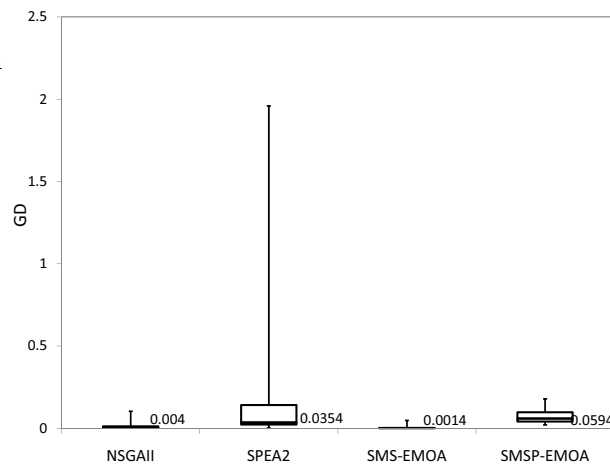

(e) GD in DTLZ3

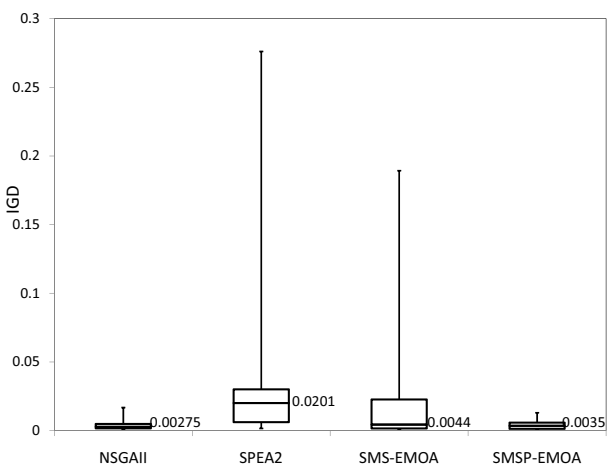

(c) IGD in ZDT4

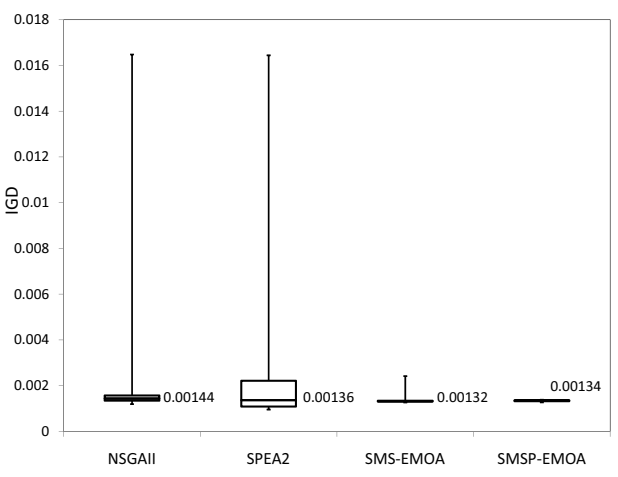

(f) IGD in DTLZ3

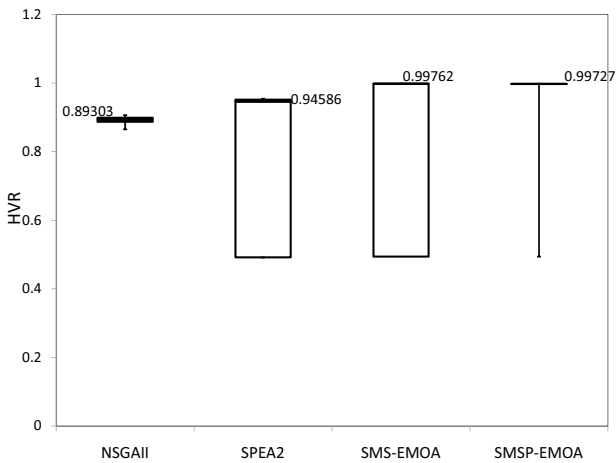

(g) HVR in DTLZ4

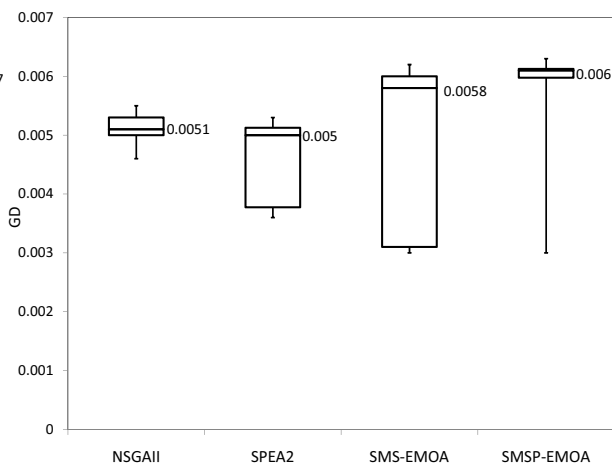

(h) GD in DTLZ4

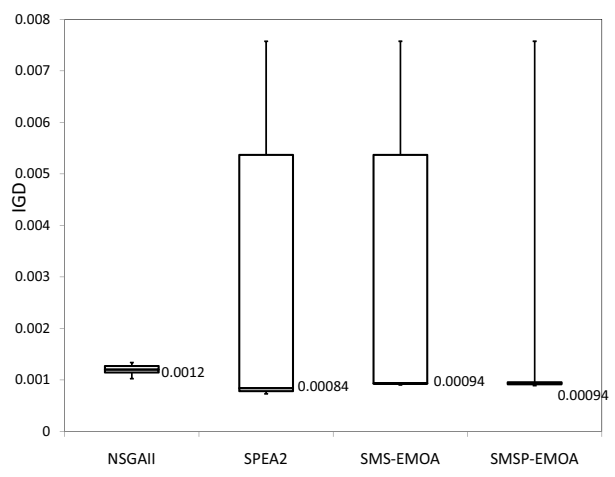

(i) IGD in DTLZ4

Figure 3: Boxplots for ZDT4, DTLZ3 and DTLZ4

\section{Convergence Velocity Analysis}

This section evaluates the convergence velocity of SMSPEMPA and other three algorithms with HVR. Figure 4 shows how four algorithms improve their HVR values as the number of function evaluations grows. SMSP-EMOA improves its HVR faster than the other three algorithms in all problems except ZDT2 and DTLZ4. Particularly, in ZDT4, ZDT6 and DLTZ3, SMSP-EMOA's convergence velocity is significantly faster than the others'. For example, in ZDT6, SMSP-EMOA yields the HVR value of 0.45 in the 7,500th function evaluation while the other three algorithms' HVR values are still zero at that time.

In ZDT2 and DTLZ4, SMSP-EMOA's convergence velocity is not the best and slower than NSGAII at the beginning of experiments. However, in ZDT2, its HVR is higher than NSGAII's at the last function evaluation. In DTLZ4, its HVR is comparable to NSGAII's at the last function evaluation.

In DTLZ2, NSGAII and SPEA2 encounter premature convergence. SMS-EMOA and SPEA2 have the same problem in DTLZ4. In contrast, SMSP-EMOA never encounters 
premature convergence. In terms of convergence velocity, SMSP-EMOA is more consistent and robust than the other three algorithms among different problems.

\section{Conclusions}

SMSP-EMOA is a new indicator-based EMOA that leverages different quality indicators in different selection operators. It uses the prospect indicator in its parent selection and the hypervolume indicator in its environmental selection. Experimental results show that SMSP-EMOA's parent selection operator complement its environmental selection operator. SMSP-EMOA outperforms SMS-EMOA and wellknown traditional EMOAs in optimality and convergence velocity without sacrificing the diversity of individuals.

Several future extensions are planned as future work. First, an extended set of experiments will be carried out to evaluate SMSP-EMOA and compare it with other algorithms in higher dimensional DTLZ problems that have more than three objectives. Another set of experiments is planned to examine SMSP-EMOA in more realistic problems such as the Traveling Salesperson Problem and the Vehicle Routing Problem. Secondly, SMSP-EMOA will be equipped with an online/offline convergence detection algorithm that determines the termination condition (i.e., the maximum number of objective function evaluations) in each experiment.

\section{REFERENCES}

[1] R. C. Purshouse and P. J. Fleming, "Evolutionary manyobjective optimization: An exploratory analysis," in Proc. IEEE Congress on Evolutionary Computation, 2003.

[2] N. Srinivas and K. Deb, "Multiobjective function optimization using nondominated sorting genetic algorithms," Evol. Computat., vol. 2, no. 3, 1995.

[3] C. C. Coello, "Evolutionary multi-objective optimization: Some current research trends and topics that remain to be explored," Front. Computat. Sci. China, vol. 3, no. 1, 2009.

[4] E. Zitzler and L. Thiele, "Multiobjective optimization using evolutionary algorithms: A comparative study," in Proc. Int'l Conference on Parallel Problem Solving from Nature, 1998.

[5] E. Zitzler, D. Brockho, and L. Thiele, "The Hypervolume Indicator Revisited: On the Design of Pareto-compliant Indicators Via Weighted Integration," in Proc. of Int'l Conference on Evolutionary Multi-Criterion Optimization, 2007.

[6] J. Bader and E. Zitzler, "HypE: An algorithm for fast hypervolume-based many-objective optimization," Evol. Computat., vol. 19, no. 1, 2011.

[7] E. Zitzler and S. Kuenzli., "Indicator-based selection in multiobjective search," in Proc. of Int'l Conference on Parallel Problem Solving from Nature, 2004.

[8] E. Zitzler, L. Thiele, and J. Bader, "SPAM: Set preference algorithm for multiobjective optimization," in Proc. of Int'l Conference on Parallel Problem Solving From Nature, 2008.
[9] K. Le and D. Landa-Silva, "Obtaining better non-dominated sets using volume dominance," in Proc. of IEEE Congress on Evol. Computat., 2007.

[10] T. Wagner, N. Beume, and B. Naujoks, "Pareto-, aggregation-, and indicator-based methods in many-objective optimization," in Proc. Int'l Conf. Evol. Multi-criterion Optimization, 2007.

[11] N. Beume, B. Naujoks, and M. Emmerich, "SMS-EMOA: Multiobjective selection based on dominated hypervolume," Eur. J. Oper. Res., vol. 181, no. 3, 2007.

[12] P. Koch, O. Kramer, G. Rudolph, and N. Beume, "On the hybridization of SMS-EMOA and local search for continuous multiobjective optimization," in Proc. the Genetic and Evolutionary Computation Conference, 2009.

[13] J.-W. Klinkenberg, M. T. M. Emmerich, A. H. Deutz, O. M. Shir, and T. Back, "A reduced-cost SMS-EMOA using kriging, self-adaptation, and parallelization," in Proc. Int'l Conference on Multiple Criteria Decision Making, 2008.

[14] H. Li, G. Casale, and T. Ellahi, "SLA-driven planning and optimization of enterprise applications," in Proc. WOSP/SIPEW Int'l Conference on Performance Engineering, 2010.

[15] E. Reehuis, "Multiobjective optimization of water distribution networks using SMS-EMOA," Leiden University, Tech. Rep. 08-12, 2008

[16] B. Naujoks and H. Trautmann, "Online convergence detection for multiobjective aerodynamic applications," in Proc. IEEE Congress on Evolutionary Computation, 2009.

[17] H. Ishibuchi, N. Tsukamoto, Y. Sakane, and Y. Nojima, "Indicator-based evolutionary algorithm with hypervolume approximation by achievement scalarizing functions," in Proc. ACM Genetic and Evolutionary Computat. Conference, 2010.

[18] K. Deb, A. Pratap, S. Agarwal, and T. Meyarivan, "A fast and elitist multiobjective genetic algorithm: NSGA-II," IEEE Trans Evol. Computat., vol. 6, no. 2, 2002.

[19] E. Zitzler, M. Laumanns, and L. Thiele, "SPEA2: Improving the Strength Pareto Evolutionary Algorithm for Multiobjective Optimization," in Evol. Methods for Design, Optimisation and Control with Application to Industrial Problems, 2002.

[20] J. Durillo, A. Nebro, and E. Alba, "The jMetal framework for multi-objective optimization: Design and architecture," in Proc. IEEE Congress on Evolutionary Computation, 2010.

[21] A. Auger, J. Bader, D. Brockhoff, and E. Zitzler, "Theory of the hypervolume indicator: Optimal $\mu$-distributions and the choice of the reference point," in Proc. of ACM Int'l Workshop on Foundations of Genetic Algorithms, 2009.

[22] K. Deb, K. Sindhya, and T. Okabe, "Self-adaptive simulated binary crossover for real-parameter optimization," in Proc. of ACM Genetic and Evol. Computat. Conference, 2007.

[23] E. Zitzler, K. Deb, and L. Thiele, "Comparison of multiobjective evolutionary algorithms: Empirical results," Evol. Computat., vol. 8, no. 2, 2000. 


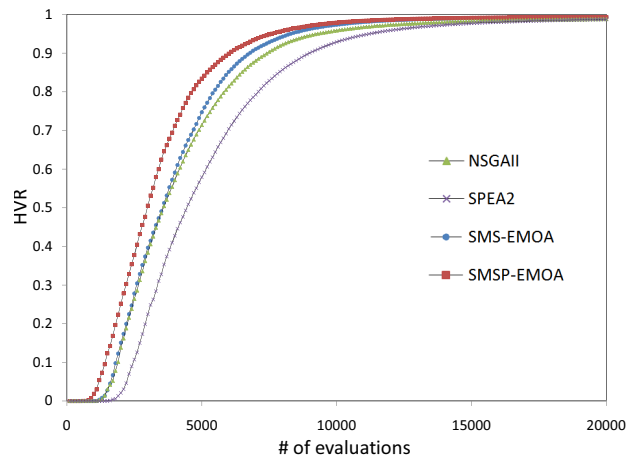

(a) ZDT1

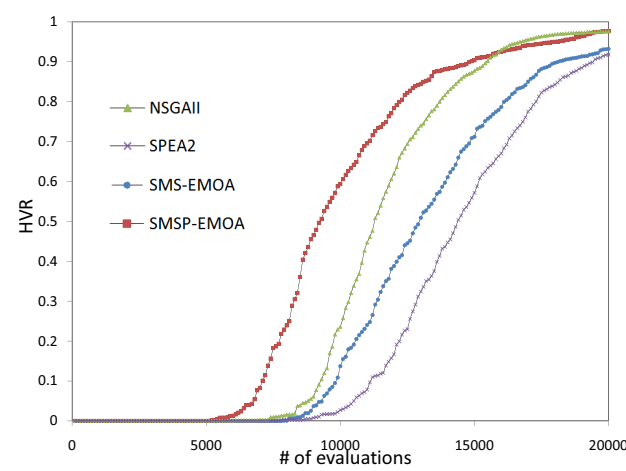

(d) ZDT4

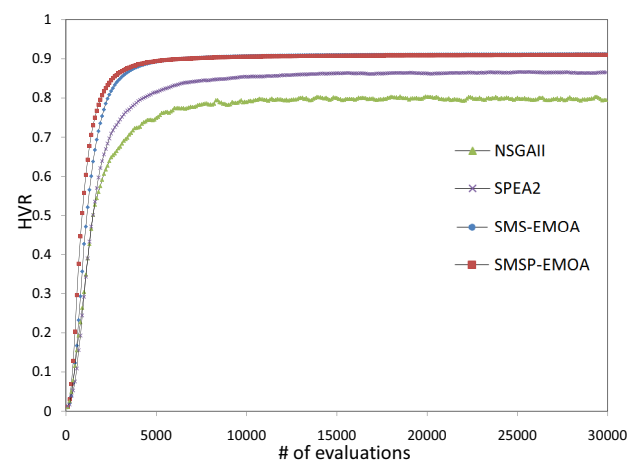

(g) DTLZ2

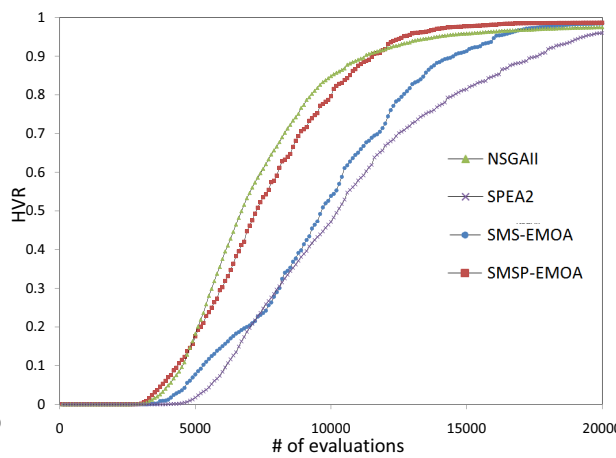

(b) ZDT2

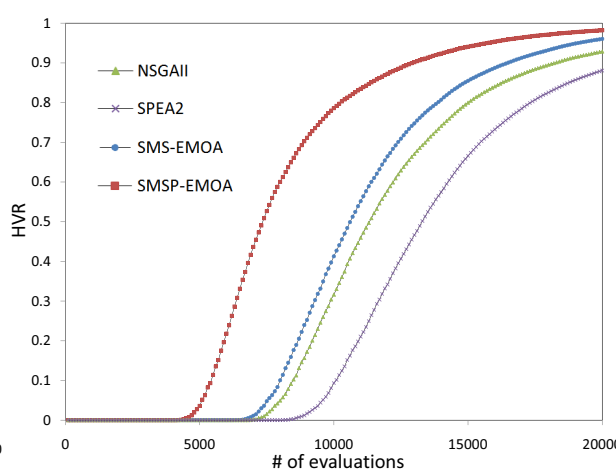

(e) ZDT6

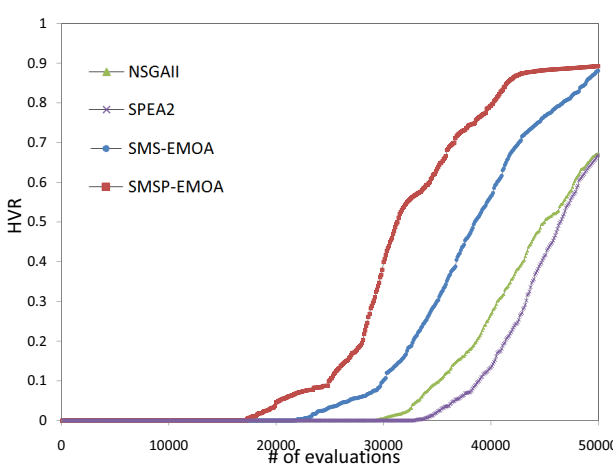

(h) DTLZ3

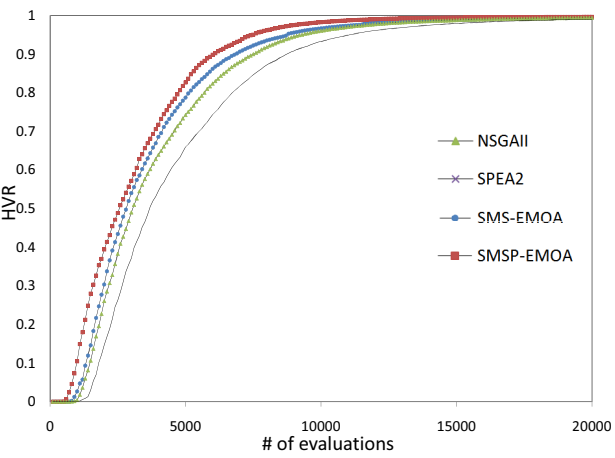

(c) ZDT3

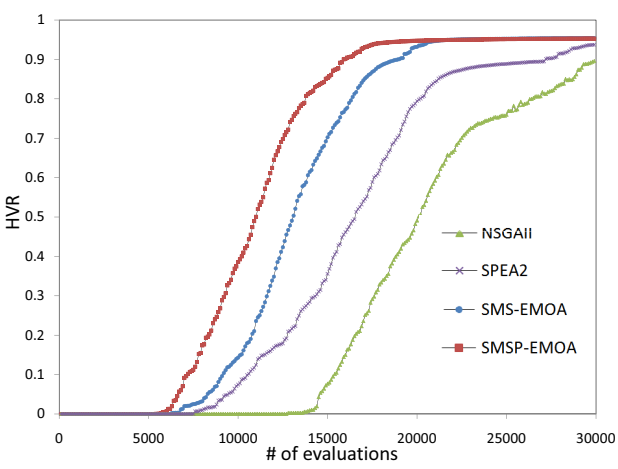

(f) DTLZ1

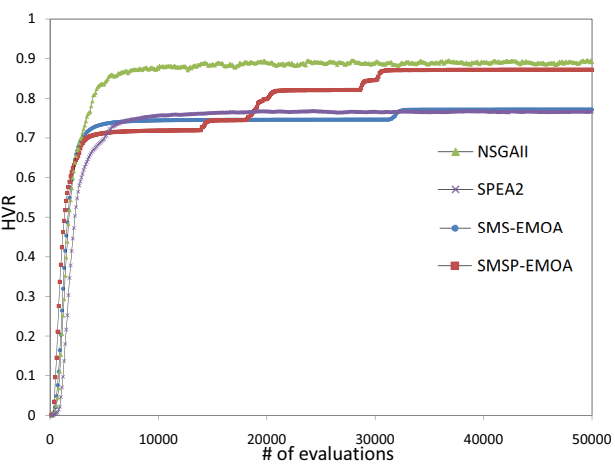

(i) DTLZ4

Figure 4: Convergence Velocity

[24] K. Deb, L. Thiele, M. Laumanns, and E. Zitzler, "Scalable test problems for evolutionary multiobjective optimization," in Evolutionary Multiobjective Optimization, A. Abraham, R. Jain, and R. Goldberg, Eds. Springer, 2005.

[25] D. A. V. Veldhuizen and G. B. Lamont, "Multiobjective evolutionary algorithm test suites," in Proc. ACM Symposium on Applied Computing, 1999.

[26] — - "Multiobjective evolutionary algorithm research: A history and analysis," in Technical Report TR-98-03, Air Force Institute of Technology, 1998. 